\title{
A prophylactic vaccine for breast cancer?
}

\author{
Christine J Watson ${ }^{1 *}$ and Barry A Gusterson ${ }^{2}$
}

\begin{abstract}
Cancer vaccines are the Holy Grail for patients and clinicians alike. The possibility that we can be vaccinated against common cancers is very appealing and the socioeconomic consequences are significant. A recent paper published in the journal Nature Medicine suggests a new approach for the development of a prophylactic vaccine for breast cancer. Their strategy was to induce mammary gland failure in mice by immunisation with an antibody specific to a milk protein that resulted in autoimmunity during lactation. This also showed some efficacy as a therapeutic vaccine. Can we look forward to the elimination of breast cancer?
\end{abstract}

Breast cancer is a very common disease, affecting approximately one in nine women in the western world at some time in their lives. In recent years, passive immunotherapy has become an effective adjunct for the treatment of HER2/neu-overexpressing breast cancers that often respond well to agents such as trastuzumab. This therapy is only effective, however, in a subset of breast cancers and patients with late-stage disease who are often immune suppressed are unlikely to respond. Furthermore, tumours can evolve to evade the immune response through regulatory $\mathrm{T}$ cells and myeloid suppressor cells $[1,2]$. There is therefore a need for a more globally effective, prophylactic vaccine.

Considerable effort has been directed towards this end, with limited success to date. A major problem with vaccine development is that the antigenic determinant ideally should be tumour specific. One such example is the glycoprotein mucin 1 (MUC-1), which is expressed by normal mammary epithelial cells. In cancer cells, however, the glycosylation pattern of MUC-1 is altered rendering it antigenic [3]. Several promising

\footnotetext{
*Correspondence: cjw53@mole.bio.cam.ac.uk

'Department of Pathology, University of Cambridge, Tennis Court Road, Cambridge CB2 1QP, UK

Full list of author information is available at the end of the article
}

MUC-1-based vaccines have been developed, although these have only been tested in a preclinical setting [4]. HER2 immunotherapy works because the density of the antigen is much higher in the cancer cells as a result of HER2 overexpression [5].

The recent development of a prophylactic vaccine for cervical cancer [6] promises to save the lives of young women worldwide, particularly where screening programmes are unavailable. Breast cancer is much more likely to affect women than cervical cancer, and so the news that a prophylactic vaccine for breast cancer had been developed caused a media frenzy and was headline news.

The cause of all this excitement was a paper published in Nature Medicine by Jaini and coworkers [7]. Their approach was based on the hypothesis that removal of an organ will provide protection against cancer developing in that organ. This seems a simple, if somewhat dramatic, approach, and the breast is the only organ that perhaps could be induced to undergo catastrophic failure without severe consequences for the individual.

With this idea in mind, a protein specific for differentiated mammary epithelial cells was selected $(\alpha-$ lactalbumin - a milk protein) and recombinant protein was used to immunise female mice. Interestingly, nonpregnant mice have few inflammatory cells in their mammary tissue but lactating mice had infiltrates of $\mathrm{T}$ cells and apparent lactation failure as pups failed to thrive, suggesting that the T cells had a cytotoxic effect. A more comprehensive follow-up of these animals would have provided reassurance that they did not suffer from detrimental effects arising from the extensive inflammation in their mammary tissue, particularly since milk proteins are expressed in the gland with every oestrus cycle.

The next step was to test whether immunisation with $\alpha$-lactalbumin could provide prophylactic protection against mammary cancer. Young mice harbouring an MMTV-Neu oncogene were therefore immunised, and tumour growth was then monitored after 8 months. Although the numbers of mice are small, the vaccine was $100 \%$ effective. It would have been worthwhile following the immunised animals for longer to conclude that they did not develop cancer later. Nevertheless, these data suggest that immunisation can provide protection from breast cancer. 
In a different tumour model - transplanted 4T1 cells tumour growth was reduced in terms of size but not delayed in onset if immunisation occurred 13 days before tumour cell inoculation. The focus of the work then turned to the potential therapeutic ability of $\alpha$ lacatalbumin immunisation to inhibit the growth of already established tumours. Inoculation of $4 \mathrm{~T} 1$ cells followed by immunisation decreased the effectiveness of the vaccine considerably, and a gap of 21 days between inoculation and immunisation resulted in no significant protection. If the experiments had been continued for longer than 32 days, it seems very likely that there would be no real difference between the immunised mice and mock-immunised mice in terms of tumour burden.

Could this be an exciting prophylactic vaccine? In comparison with the vaccine for cervical cancer, which is primarily given to teenage girls, we suspect that most young women would not be willing to avoid lactation as the authors suggested - this is a natural consequence of pregnancy. Is it likely that women would not breast feed their infants in order to avoid a cancer that they have a $10 \%$ chance of developing 30 years later? This approach is therefore unlikely to be adopted as a prophylactic vaccine. Could it be useful for therapeutic purposes? That depends on the expression of $\alpha$-lactalbumin by breast cancer cells, particularly the cancer-initiating (stem) cells, since eliminating the bulk of the tumour and not the cancer stem cells will not prevent recurrence of the tumour.

Whether $\alpha$-lactalbumin is expressed in human breast cancers is not clear as the mRNA has not been identified in breast cancer samples [8] and - as shown in this key publication by Hall and colleagues - there is a peptide that shares antigenic determinants with $\alpha$-lactalbumin and with which antibodies to $\alpha$-lactalbumin immunoreact. Bailey and colleagues, using a well-characterised antibody, could not detect $\alpha$-lactalbumin in human breast cancers [9], whilst it was present in pregnant breast and in lactational foci seen in normal virgin women taking oral contraceptives. Current data on $\alpha$ lactalbumin expression are based on contradictory data that are now nearly three decades old. There is sufficient uncertainty regarding the expression of $\alpha$-lactalbumin in both the normal breast and breast cancers to question the validity of considering $\alpha$-lactalbumin as a vaccine target in humans.

It is unfortunate that this preliminary study should be the subject of such unrealistic press coverage in such an emotive arena.

\section{Competing interests}

The authors declare that they have no competing interests.

\section{Author details}

'Department of Pathology, University of Cambridge, Tennis Court Road, Cambridge CB2 1QP, UK. ${ }^{2}$ Department of Pathology, Institute of Cancer Sciences, Western Infirmary, Glasgow G61 2RN, UK.

Published: 31 August 2010

\section{References}

1. Larmonier N, Janikashvili N, LaCasse CJ, Larmonier CB, Cantrell J, Situ E, Lundeen T, Bonnotte B, Katsanis E: Imatinib mesylate inhibits CD4+ CD25+ regulatory $T$ cell activity and enhances active immunotherapy against $\mathrm{BCR}^{-} \mathrm{ABL}^{-}$tumors. J Immunol 2008, 181:6955-6963.

2. Heithoff DM, Enioutina EY, Bareyan D, Daynes RA, Mahan MJ: Conditions that diminish myeloid-derived suppressor cell activities stimulate crossprotective immunity. Infect Immun 2008, 76:5191-5199.

3. Carr-Brendel V, Markovic D, Ferrer K, Smith M, Taylor-Papadimitriou J, Cohen EP: Immunity to murine breast cancer cells modified to express MUC-1, a human breast cancer antigen, in transgenic mice tolerant to human MUC-1. Cancer Res 2000, 60:2435-2443.

4. Yuan S, Shi C, Liu L, Han W: MUC1-based recombinant Bacillus CalmetteGuerin vaccines as candidates for breast cancer immunotherapy. Expert Opin Biol Ther 2010, 10:1037-1048.

5. Baxevanis CN, Voutsas IF, Gritzapis AD, Perez SA, Papamichail M: HER-2/neu as a target for cancer vaccines. Immunotherapy 2010, 2:213-226.

6. Stanley M, Gissmann L, Nardelli-Haefliger D: Immunobiology of human papillomavirus infection and vaccination - implications for second generation vaccines. Vaccine 2008, 26(Suppl 10):K62-K67.

7. Jaini R, Kesaraju P, Johnson JM, Altuntas CZ, Jane-Wit D, Tuohy VK: An autoimmune-mediated strategy for prophylactic breast cancer vaccination. Nat Med 2010, 16:799-803.

8. Hall L, Craig RK, Davies MS, Ralphs DNL, Campbell PN: a-Lactalbumin is not a marker of human hormone-dependent breast cancer. Nature 1981, 290:602-604.

9. Bailey AJ, Sloane JP, Trickey BS, Ormerod MG: An immunocytochemical study of a-lactalbumin in human breast tissue. J Pathol 1982, 137:13-23.

doi: $10.1186 /$ bcr 2628

Cite this article as: Watson CJ, Gusterson BA: A prophylactic vaccine for breast cancer? Breast Cancer Research 2010, 12:310. 